\title{
RESOURCE ALLOCATION, HYPERPHAGIA, AND COMPENSATORY GROWTH IN JUVENILES
}

\author{
William S. C. Gurney, ${ }^{1,3}$ Wayne Jones,,${ }^{1}$ A. Roy Veitch, ${ }^{1}$ and Roger M. Nisbet ${ }^{2}$ \\ ${ }^{1}$ Department of Statistics and Modelling Science, University of Strathclyde, Livingstone Tower, Richmond Street, \\ Glasgow, Scotland G1 1XT, UK \\ ${ }^{2}$ University of California, Santa Barbara, California 95106 USA
}

\begin{abstract}
Many organisms exploit highly variable food supplies and, as an adaptation to such conditions, show elevated growth during recovery from starvation. In some species this response enables starved and re-fed individuals to outpace those growing continuously.

The main engine of compensatory growth is a relative increase in food ingestion as a reaction to poor nutritional condition. We use a series of mathematical energy-budget models to investigate the interaction between the mechanisms that control such hyperphagia and those that control internal allocation, with the aim of identifying those strategies that permit overcompensation.

We find that hyperphagia alone normally produces weak compensation and can never result in overcompensation. When combined with internal allocation, which routes a fixed fraction of net production to reserves, a strong compensatory response becomes the norm, and overcompensation is frequent.
\end{abstract}

Key words: compensatory growth; energy-budget models; hyperphagia; juvenile growth; overcompensation; resource allocation; starvation recovery.

\section{INTRODUCTION}

In the natural environment many organisms exploit food supplies whose spatial, temporal, or seasonal variability implies significant periods of near or actual famine. As an adaptation to such a lifestyle, many organisms exhibit faster growth during recovery from starvation than during constant exposure to the same food environment. Such growth compensation has been observed in invertebrates (Bradley et al. 1991) as well as fish (Weatherly and Gill 1981), mammals (Kennedy 1953) and birds (Wilson and Osbourne 1960).

In some species, compensation can be so intense that starved and re-fed individuals exhibit a higher average growth rate than those fed continuously. This phenomenon has been observed in fish (e.g., Miglavs and Jobling 1989b) and poultry (Yu et al. 1990). It has clear ecological implications and has attracted considerable applied-research interest because of its potential impact on feed-lot husbandry (e.g., Hayward et al. 1997).

In order to increase its growth rate an individual must either raise its resource intake or decrease its metabolic costs. Although some workers have reported reduced basal metabolism (O'Connor et al. 2000) and/or increased conversion efficiency (Russel and Wooton 1992) during recovery, the effect is usually small and is often also short lived (Miglavs and Jobling 1989a). By contrast, large increases in ingestion rate under recovery conditions (hyperphagia) have been demonstrated in a variety of mammals (Weigle 1994, Blum

Manuscript received 28 August 2002; revised and accepted 3 February 2003. Corresponding Editor: M. G. Neubert.

${ }^{3}$ E-mail: bill@stams.strath.ac.uk
1997, Friedman 1998) and in salmonid fish (Miglavs and Jobling 1989b, Metcalfe and Thorpe 1992, Jobling and Johansen 1999).

A connection between reduced adiposity and increased ingestion, now generally known as the "lipostatic model," was first proposed for rats by Kennedy (1953). For a number of mammalian species both the existence of compensatory hyperphagia and the underlying biochemical mechanism are now well established (Blum 1997). A number of workers (Bull and Metcalf 1996, Jobling and Johansen 1999) have suggested that a similar mechanism operates in salmonid fish.

A theoretical understanding of the dynamics of enhanced growth requires a description of the mechanisms that control an individual's energy budget. Kooijman (1993) has developed a group of "dynamic energy budget" models that are capable of explaining a wide variety of growth and body-size scaling effects (e.g., Nisbet et al. 2000). Muller and Nisbet (2000) examined the behavior of these models in fluctuating environments and showed that under certain conditions such environmental fluctuations can produce enhanced growth. However, the underlying mechanism identified by Muller and Nisbet (2000) does not resemble that suggested by the above experimental results, and the total predicted enhancement is clearly insufficient to imply overcompensation.

The work reported here developed from a more closely focused theoretical study of growth compensation reported by Broekhuisen et al. (1994). Their dynamic description, which assumed both compensatory hyperphagia and cost reduction, postulated that the primary purpose of compensation was to restore nutri- 
tional condition and thus routed additional ingestate primarily to reserves. More recent work on this model (Jones 2001) has shown that it is systematically incapable of predicting overcompensation.

In this paper we investigate a model whose basic structure is very similar to that of Broekhuisen et al. (1994) but whose description of hyperphagia and resource allocation are designed to allow plasticity of behavior between resource recovery and enhanced overall growth. We take particular note of the review of lipostasis in fish by Jobling and Johansen (1999), who point out that individuals who replenish their reserves rapidly during recovery will exhibit much briefer periods of hyperphagia than individuals whose recovery strategy routes additional assimilate preferentially to structure.

Our particular concern is to identify control mechanisms that permit strong compensation. We show that hyperphagia operating alone produces weak compensation and incomplete catch-up. However, when it operates in combination with an allocation control strategy that routes ingestate preferentially to structure at high feeding rates, complete catch-up becomes the norm.

Finally, we note that an allocation control mechanism designed to make the nutritional condition of an animal growing in constant conditions independent of its environment would have precisely the properties required to amplify hyperphagia. A corollary of such a control mechanism is that, under constant environmental conditions, a fixed fraction of net somatic production is allocated to increasing the overall size of the animal.

\section{The Model}

\section{State variables}

Our model is an extension of that proposed by Jones et al. (2002) to explain field observations of seasonal growth in salmon parr, which was in turn a development of one used by Broekhuisen et al. (1994) to explain compensatory growth in laboratory salmonid populations subject to cyclic ration variations. We follow them in using carbon mass as our currency. The tight coupling between this and energy content (e.g., Carter et al. 1992) implies that our model is a dynamic energybudget model in the sense of Kooijman (1993).

Following both predecessors, we divide the total carbon mass of a reproductively inactive individual into two components. Reserves $(R)$ represent the part that can be mobilized to meet basal metabolic costs under starvation conditions and are identified with fatty deposits and remobilizable parts of the musculature. Structure $(S)$ represents the part that cannot be remobilized and is identified with skeletal, circulatory, and nervous tissue.

Both earlier models used structural and reserve carbon masses $(S, R)$ to characterize the individual's state and assayed its nutritional condition from their ratio.
We vary this approach and characterize individual state by the combination of structural carbon $(S)$ and the fraction $(X)$ of total carbon held as reserves, i.e.,

$$
X \equiv \frac{R}{R+S} .
$$

The individual's reserve and total carbon masses are related to our chosen state description $(S, X)$ by

$$
\begin{aligned}
R & =\frac{S X}{1-X} \\
R+S & =\frac{S}{1-X} .
\end{aligned}
$$

\section{Assimilation and maintenance}

As advocated by Gurney and Nisbet (1998) we divide metabolic costs into those that are proportional to food uptake rate and those that are independent of it. We focus on growth rather than environmental impact, so we use $A$ to denote the resource acquisition rate net of feeding-rate-dependent costs. We shall refer to feedingrate-independent costs as "basal maintenance" $(M)$, because they may contain an activity element in addition to basal metabolism.

Following Jones et al. (2002) we assume that $A$ scales allometrically with structural carbon, while $M$ scales linearly with total carbon, so that

$$
\begin{aligned}
A & =\alpha S^{\beta} \\
M & =\frac{\mu S}{1-X} .
\end{aligned}
$$

We shall regard the assimilation rate power, $\beta$, as constant, but the basal maintenance rate scale, $\mu$, and the assimilation rate scale, $\alpha$, must be environment dependent. At a minimum, both depend on the environmental temperature, and $\alpha$ additionally depends on food availability.

\section{Diet and allocation}

Many dynamic energy budget (DEB) models regard food and energy as synonymous, but in this study we divide the ingestate stream into two components. One, which we shall call "fat," can only be burned to meet metabolic costs or stored to be used for that purpose at some future date. The other, which we shall call "protein," can either be used to build new structure or broken down and burned to meet metabolic costs.

If we denote the fraction of "protein" in the net assimilate stream by $p$, then it is clear that the maximum rate at which carbon could be committed to new structure is $p A$. However, if the rates of assimilation and basal maintenance are comparable, then allocating to structure all assimilate that could be so allocated will result in reserves being metabolized to meet basal metabolic requirements. Such a policy may be tenable 


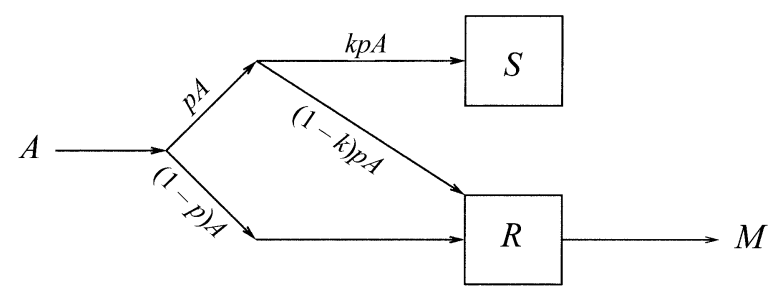

FIG. 1. Schematic diagram of energy allocation. $A=$ resource acquisition rate (net of feeding-rate-dependent costs), $p=$ the "protein" (non-fat) fraction in the net assimilation stream, $k=$ the fraction of the assimilated "protein" stream committed to new structure, $R=$ reserves (the part of carbon mass that can be mobilized to meet basal metabolic costs), $S$ $=$ structure (the part of carbon mass that cannot be remobilized [skeletal, circulatory, and nervous tissue]), and $M=$ basal maintenance (feeding-rate-independent costs).

over short periods but will result in reserve exhaustion and eventual death if pursued indefinitely.

To avoid such an outcome, the individual must be able to allocate some of the protein component of the assimilate stream to meet metabolic costs. Our model recognizes this possibility by assuming that a fraction $k$ of this component is actually committed to new structure, while the remainder is allocated to reserves.

This allocation scheme, which is illustrated in Fig. 1 , carries the implication that an individual's structure does not shrink during a bout of starvation. The accuracy of this view clearly depends on the definition of structural tissue. However, for salmon parr Jones et al. (2002) have argued that structural mass is a surrogate for body length, which is observed to stay constant during starvation-induced mass loss. Similar effects have been observed in other species (for example, Daphnia; McCauley et al. 1990).

\section{Dynamics}

We can see from Fig. 1 that the time rate of change of structural carbon is

$$
\frac{d S}{d t}=k p A
$$

To calculate the rate of change of the reserve fraction $(X)$ we first note that Fig. 1 implies that the rate of change of total reserve carbon $(R)$ is

$$
\frac{d R}{d t}=A-M-k p A .
$$

Differentiating the definition of reserve fraction (Eq. 1) and substituting from Eqs. 5, 6, and 7 then yields

$$
\frac{d X}{d t}=(1-X)\left[\frac{A}{S}(1-k p-X)-\mu\right] \text {. }
$$

\section{Development in a Constant Environment}

Under constant environmental conditions the assimilation and basal maintenance rate scales $(\alpha, \mu)$ and the dietary proportion of protein $(p)$ are constants.

\section{Starvation}

Under starvation conditions the net assimilation rate scale $(\alpha)$ is zero-implying that the net assimilation rate $(A)$ is also zero. When $A=0$, Eq. 6 tells us that structural carbon remains constant, while the explicit solution of Eq. 8, namely,

$$
X(t)=1-[1-X(0)] e^{\mu t}
$$

shows us that reserve fraction $(X)$ goes to zero in finite time. This happens because basal maintenance is proportional to total carbon, which becomes indistinguishable from structural carbon (and hence constant) as reserves are exhausted.

\section{Growth with constant allocation}

Our first examination of growth in a constant environment concerns a notional organism that cannot vary the proportion of protein committed to new structure, so $k$ has a constant value, $k_{0}$. When $\alpha>0$ and $k$ and $p$ are constants, we can restate the reserve dynamics (Eq. 8) as

$$
\frac{d X}{d t}=\left[\frac{A(1-X)}{S}\right]\left(X^{*}-X\right)
$$

where

$$
X^{*} \equiv 1-k_{0} p-\frac{\mu S}{\alpha S^{\beta}} .
$$

If $\beta=1$, Eq. 11 shows that $X^{*}$ is a constant. Eq. 10 then shows that $X^{*}$ is an attracting stationary state providing it is positive (i.e., if $\alpha>\mu /\left(1-k_{0} p\right)$ ). In this special case, we conclude that if the food supply is large enough to permit growth, then an individual growing under constant environmental conditions asymptotically approaches a constant reserve fraction given by Eq. 11 .

In fact, the transient leading to the steady-state reserve fraction state is relatively rapid (see Fig. 2a). Thus, except for a very short period after a given condition is established, the value of $X^{*}$ given by Eq. 11 will be a good measure of the nutritional condition of individuals with $\beta=1$ growing in a constant environment.

When $\beta$ is not unity, $X^{*}$ is no longer a constant. However, we expect $\beta$ to be in the range $[1 / 2,1]$, so $X^{*}$, which decreases as $S^{1-\beta}$, will change quite slowly with $S$. In this case we expect that after a brief transient the value of $X$ should remain close to $X^{*}$; this is confirmed by Fig. $2 b$ and $c$.

In recognition of its role as a measure of the nutritional condition of an individual growing in a constant environment, we shall henceforth refer to $X^{*}$ as the "quasi-stationary reserve fraction." We see from Eq. 11 that this quantity increases with increasing assimilation rate scale $(\alpha)$ and hence with increasing food availability. 

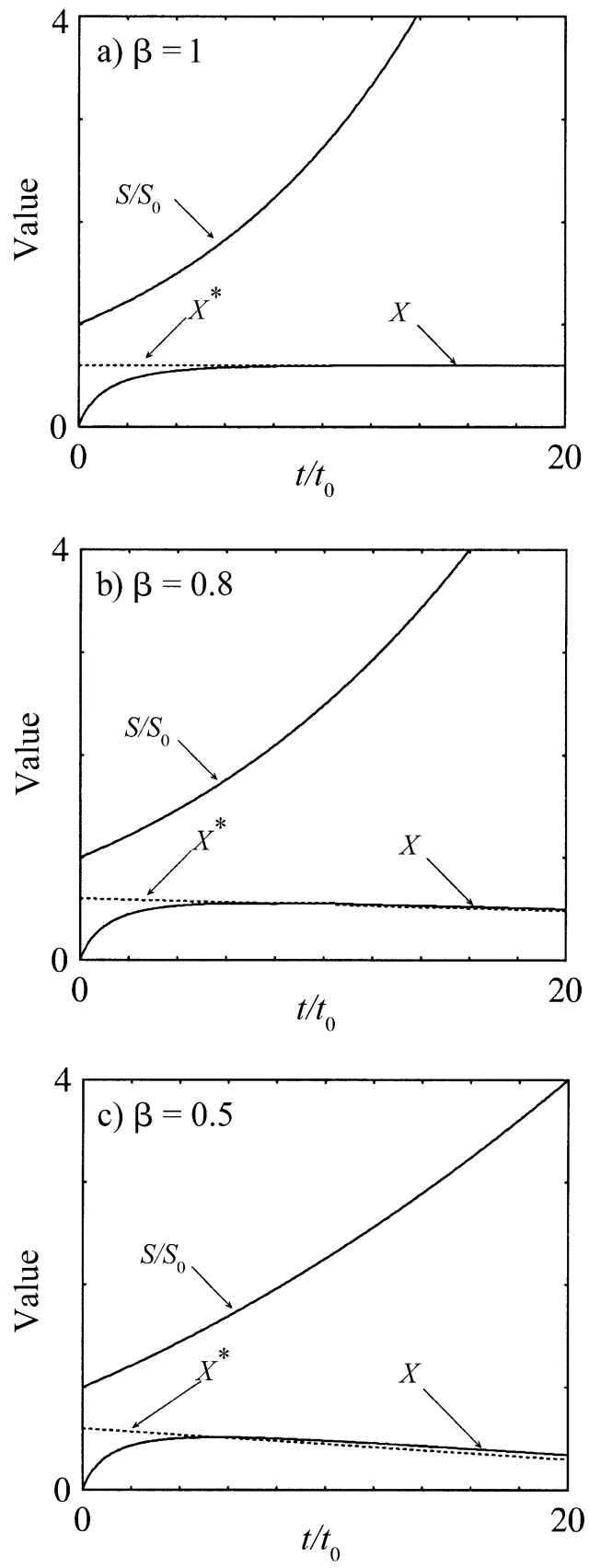

FIG. 2. Constant food growth with varying assimilation allometry $(\beta)$. Structure and time scales, $S_{0}$ and $t_{0}$, respectively, are $S_{0}=S(0)$ and $t_{0}=1 /\left(\alpha S_{0}^{\beta-1}\right)$. Solid lines show trajectories of $S / S_{0}$ and $X$ as marked. Dotted line shows $X^{*}$ (Eq. 11). Parameters are $\mu t_{0}=0.3, k_{0} p=0.1$.

\section{Controlling protein allocation}

Because the animal described in the previous section allocates a fixed fraction of its protein intake to basal metabolism it achieves less structural growth than it could at high feeding rates and fails to maintain a minimal level of reserves at low feeding rates. To ameliorate this effect the animal must vary $k$, the fraction of the protein component $(p)$ committed to new structure, so that the fraction allocated to structure increases with the net assimilation rate $(A)$.

If the organism's target is to maintain its reserve fraction at (say) $X_{\mathrm{d}}$ then Eq. 11 shows that it needs to continuously vary the fraction of ingested protein allocated to structural growth $(k)$ so that at all times $k$ $=k_{\mathrm{c}}$, where

$$
k_{\mathrm{c}} \equiv \frac{1}{p}\left[1-X_{\mathrm{d}}-\frac{\mu S}{A}\right] .
$$

However, since $k$ must lie in the range $[0,1]$, the nearest achievable approximation to this control behavior would have

$$
k= \begin{cases}0 & \text { if } 1 \geq \mu S / A>1-X_{\mathrm{d}} \\ k_{\mathrm{c}} & \text { if } 1-X_{\mathrm{d}} \geq \mu S / A>1-X_{\mathrm{d}}-p \\ 1 & \text { if } 1-X_{\mathrm{d}}-p \geq \mu S / A>0 .\end{cases}
$$

This results in a quasi-stationary reserve fraction given by

$$
X^{*}= \begin{cases}1-\mu S / A & \text { if } 1 \geq \mu S / A>1-X_{\mathrm{d}} \\ X_{\mathrm{d}} & \text { if } 1-X_{\mathrm{d}} \geq \mu S / A>1-X_{\mathrm{d}}-p \\ 1-\mu S / A-p & \text { if } 1-X_{\mathrm{d}}-p \geq \mu S / A>0\end{cases}
$$

which we illustrate, together with the attainable value of $k$, in Fig. 3.

The restriction that $k$ must lie in the interval $[0,1]$ implies that the animal can only hold its quasi-stationary reserve fraction at $X_{\mathrm{d}}$ over a range of assimilation rates defined by

$$
1-X_{\mathrm{d}}-p \leq \frac{\mu S}{A} \leq 1-X_{\mathrm{d}}
$$

Values of $A$ below the control range $\left(\mu S / A>1-X_{\mathrm{d}}\right)$ imply that all protein is metabolized to meet basal maintenance and the reserve ratio sinks until the books balance. When $A<\mu S$ the total income is insufficient even to meet the basal maintenance requirements of the structural carbon mass and reserves are depleted until the animal dies.

When $A$ is inside the control range the animal allocates just enough protein to reserves to hold the reserve fraction at the target level. The asymptotic rate of structural growth, $(d S / d t)^{*}$, is then

$$
\left(\frac{d S}{d t}\right)^{*}=\left(1-X_{\mathrm{d}}\right)\left[A-\frac{\mu S}{1-X_{\mathrm{d}}}\right] \text {. }
$$

Comparing Eq. 16 and 5 shows us that $\mu S /\left(1-X_{\mathrm{d}}\right)$ is the total expenditure on basal maintenance when $X$ $=X_{\mathrm{d}}$, so the term in square brackets in Eq. 16 represents the asymptotic value of the net production rate. We conclude that in this regime the effect of the control mechanism is to allocate a fixed fraction of net production to structure. 

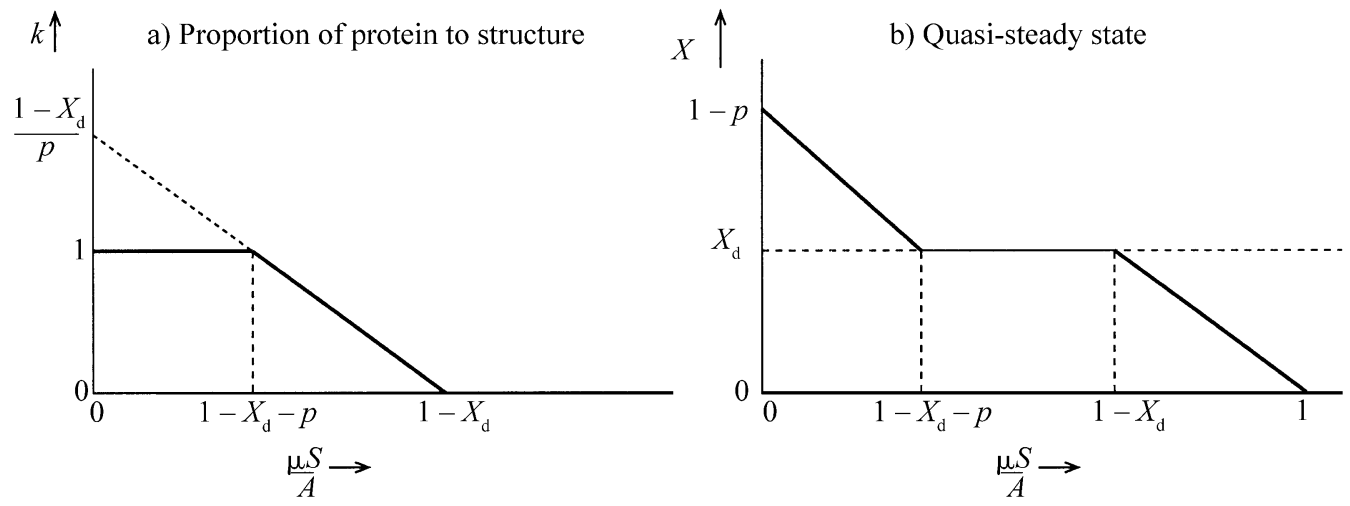

FIG. 3. Controlling $k$ to keep $X^{*}$ constant. (a) The solid line shows the attainable value of $k$ against $\mu S / A$. (b) The solid line shows achieved value of $X^{*}$ against $\mu S / A$.

When $A$ is above the control range $\left(\mu S / A<1-X_{\mathrm{d}}\right.$ $-p$ ) the animal allocates the maximum possible fraction $(p)$ of its assimilate stream to structure but cannot achieve enough structural growth to hold the reserve fraction at $X_{\mathrm{d}}$. Consequently, unless it rejects some proportion of its fat intake (a phenomenon unrepresented in this model) its quasi-stationary reserve fraction rises above $X_{\mathrm{d}}$.

\section{Hyperphagia and Growth Compensation}

Compensatory growth requires that individuals in poor nutritional condition either increase their food uptake or reduce their maintenance costs.

O'Connor et al. (2000) have shown that the basal metabolic rates of one-month-starved juvenile salmon are slightly $(\sim 20 \%)$ below those of well-fed controls. A number of experimenters have reported increases in food-conversion efficiency in the early part of refeeding experiments on fish (e.g., Russel and Wooton 1992), but these effects seem to be both small and confined to the first 14 days of refeeding (Miglavs and Jobling 1989a).

The lipostatic model proposed by Kennedy (1953) postulated that decreases in the adiposity of rats elicited compensatory increases in food intake (hyperphagia). Significant hyperphagic effects have now been demonstrated in both mammals (Weigle 1994, Blum 1997, Friedman 1998) and salmonid fish (Miglavs and Jobling 1989b, Metcalfe and Thorpe 1992, Jobling and Johansen 1999).

The compensatory-growth model proposed by Broekhuisen et al. (1994) incorporated both appetite increase and cost decrease in response to poor nutritional condition. However, the experimental evidence cited here seems to show that any maintenance cost reductions that do occur are sufficiently small and short lived that they cannot be the major contributor to compensatory growth.

In this study we regard the maintenance rate scale $(\mu)$ as a constant and concentrate on the role of lipostatic changes in food uptake. Specifically we extend our representation of assimilation (Eq. 4) by defining a hyperphagia factor $\lambda(X)$, and writing the net assimilation rate, $A$, as

$$
A=\lambda(X) \alpha S^{\beta} .
$$

To incorporate the lipostatic hypothesis, we need $\lambda$ to decrease monotonically with rising reserve fraction, crossing the value unity when $X$ is at some desired value, say $X_{\mathrm{d}}$. Many functions have this property, but one that turns out to be particularly convenient in the present context, and that we shall therefore adopt, is

$$
\lambda=\frac{X_{\mathrm{d}}}{X} .
$$

So long as the uptake rate scale $\alpha$ is not zero, Eq. 18 formally permits unlimited increases in uptake rate as $X$ falls. In reality, few individuals will survive the loss of $>95 \%$ of their reserves, which corresponds to a change in reserve fraction, and hence appetite, of about an order of magnitude.

\section{Constant allocation}

Our first variant of this model assumes that the fraction of protein allocated to structure $(k)$ has a constant value, $k_{0}$, thus allowing us to explore the effect of the control mechanism described by Eq. 18 operating in isolation. In this case the reserve fraction dynamics (Eq. 8) can be rewritten as

$$
\frac{d X}{d t}=\frac{1-X}{X}\left(\alpha X_{\mathrm{d}} S^{\beta-1}+\mu\right)\left(X^{*}-X\right)
$$

where the quasi-stationary reserve fraction is given by

$$
X^{*}=\left(1-k_{0} p\right)\left[\frac{\alpha X_{\mathrm{d}} S^{\beta}}{\alpha X_{\mathrm{d}} S^{\beta}+\mu S}\right] .
$$

From this we see that the appetite control described by Eq. 18 enables the individual to grow in any environment that results in a positive value of $\alpha$. The resulting quasi-stationary reserve fraction increases with increasing $\alpha$ (i.e., food availability and temper- 

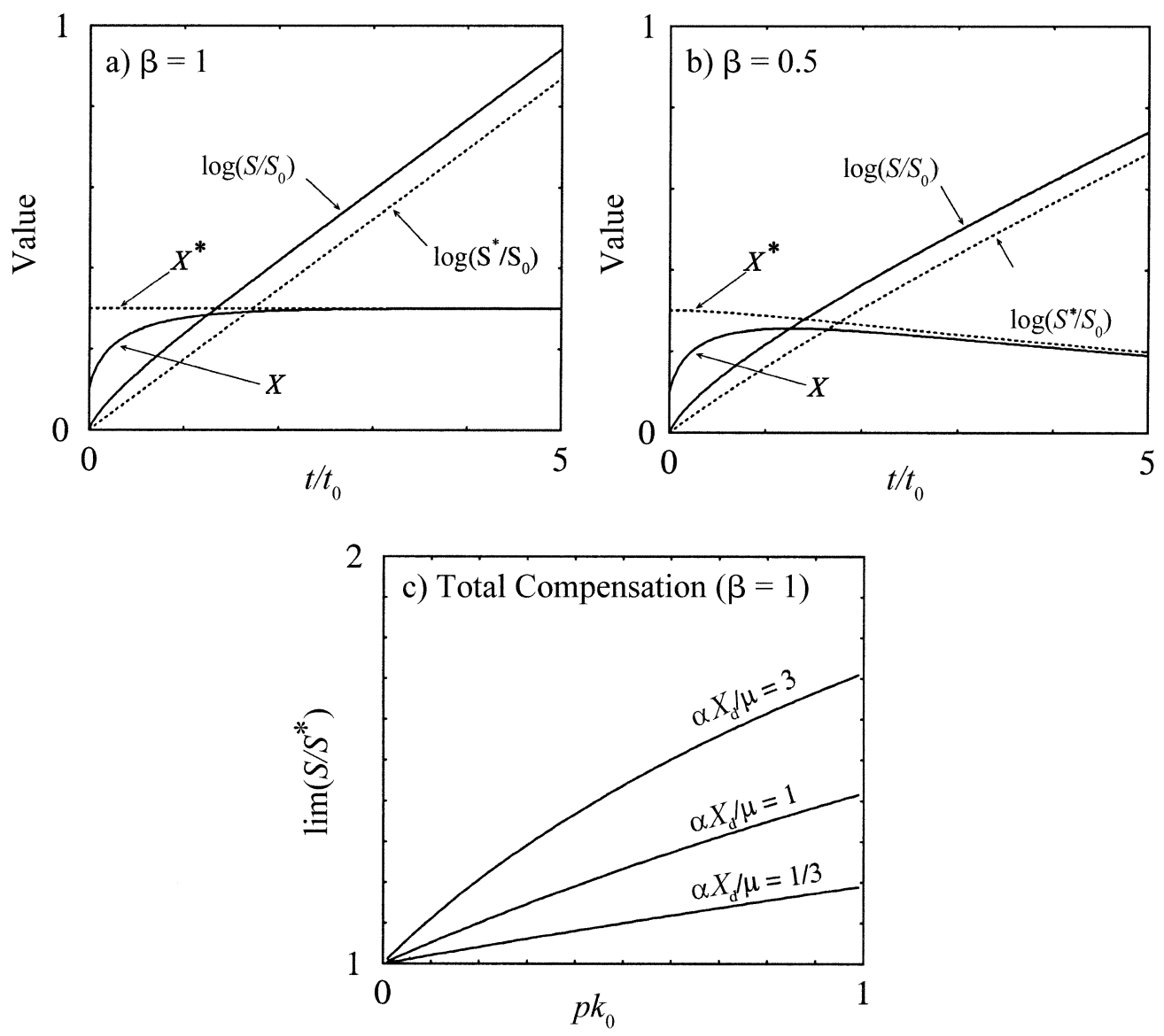

FIg. 4. Compensatory growth with constant allocation. Panels (a) and b) present solutions of Eqs. 19 and 21 for individuals starting with $X=X^{*}$ (dotted line) and $X=X^{*} / 3$ (solid line). Parameters are $\mu t_{0}=0.3, X_{\mathrm{d}}=0.5, p k_{0}=0.4$. Structure and time scales are $S_{0}=S(0)$ and $t_{0}=1 /\left(\alpha S_{0}^{\beta-1}\right)$. (c) Total compensation calculated from Eq. (24) for individuals with $\beta=1$ and $X_{0}=X^{*} / 3$, as a function of $p k_{0}$ with values of $X_{\mathrm{d}} / \mu t_{0}$ (equivalent to $\alpha X_{\mathrm{d}} / \mu$ ) as marked.

ature) and decreases (slowly) with $S$ provided the assimilation scale $\beta<1$.

By combining Eqs. 6, 17, and 18 we see that an individual with structure $S$ and reserve fraction $X$ produces new structure at a rate

$$
\frac{d S}{d t}=\frac{k_{0} p \alpha X_{\mathrm{d}} S^{\beta}}{X}
$$

which, when $X=X^{*}$, becomes

$$
\left(\frac{d S}{d t}\right)^{*}=\left(\frac{p k_{0}}{1-p k_{0}}\right)\left(\alpha X_{\mathrm{d}} S^{\beta}+\mu S\right)
$$

thus showing that the asymptotic structural growth rate increases with $\alpha$ (and hence with temperature and food availability).

Equations 21 and 22 also show that any individual whose reserve fraction $(X)$ is below the quasi-stationary value for its current environment will have a structural growth rate higher than the asymptotic value for an individual of that size in that environment.

This implies that on re-alimentation after a bout of starvation an individual will exhibit enhanced values of both assimilation and structural growth relative to a control maintained in the final environment throughout. This period of compensatory growth will last until the reserve ratio has returned to its quasi-stationary value. Since $X^{*}$ increases with $\alpha$ (and hence with food availability and temperature) an individual subjected to a rapid enhancement of environmental quality will exhibit a similar period of compensatory growth.

We illustrate this behavior in the upper frames of Fig. 4. Each shows two numerical solutions of Eq. 19 and 21 , the first representing a control individual raised in the experimental environment, and the second representing an individual transfered into that environment from a poorer one. At $t=0$ both individuals have the same structural carbon $\left(S_{0}\right)$ but the control's reserve fraction is at the appropriate quasi-stationary value $\left(X^{*}\right)$ while that of the transferred individual is set to one third of this value.

As we would expect, the transferred individual's reserve fraction eventually returns to quasi-stationarity, but while it is depressed the hyperphagic response described by Eq. 18 implies an enhanced rate of structural 
growth. Thus, although the two individuals eventually follow parallel growth trajectories, the transferred individual remains permanently larger than the control.

In the special case where assimilation is linear in $S$ (that is, $\beta=1$ ) we can obtain an analytic expression for the asymptotic size ratio resulting from a bout of compensatory growth. In the Appendix we show that two individuals with the same initial structure, but with initial reserve fractions $X_{0}$ and $X^{*}$ respectively, will have structural carbon masses $S(t)$ and $S^{*}(t)$ whose ratio tends asymptotically to a value we refer to as the "asymptotic compensation ratio," $C_{\infty}$, given by

$$
C_{\infty} \equiv \lim _{t \rightarrow \infty}\left[\frac{S(t)}{S^{*}(t)}\right]=\left[\frac{1-X_{0}}{1-X^{*}}\right]^{p k_{0} /\left(1-p k_{0}\right)} .
$$

To explore the parameter dependence of observed compensation in an experiment where $X_{0}$ is a fixed fraction of the quasi-stationary value, we must take account of the way $X^{*}$ varies (Eq. 20). If $\epsilon$ represents the ratio $X_{0} / X^{*}$, then we can rewrite Eq. 23 as

$$
C_{\infty}=\left[1+\frac{(1-\varepsilon)\left(1-p k_{0}\right)}{\left(\mu / \alpha X_{\mathrm{d}}\right)+p k_{0}}\right]^{p k_{0} /\left(1-p k_{0}\right)}
$$

which we plot, as a function of the controlling parameter groups $p k_{0}$ and $\alpha X_{\mathrm{d}} / \mu$, in Fig. 4c. From this figure, we see that compensation increases with $\alpha$ (environmental quality) as well as with the product of the dietary proportion of protein $(p)$ and the proportional allocation to structural growth $\left(k_{0}\right)$. This latter effect occurs because raising the product $p k_{0}$ increases the structural growth rate and decreases the reserve recovery rate, thus prolonging the period of hyperphagia.

Eq. 23 and 24 are only exact when assimilation is linear in $S(\beta=1)$. However, Fig. $4 \mathrm{a}$ and $\mathrm{b}$ show that the asymptotic size ratio when $\beta<1$ is comparable to, but slightly smaller than, that exhibited by an otherwise identical model with $\beta=1$. This is a universal feature of our simulations and it seems intuitively reasonable that it is a general property. We thus conjecture that the asymptotic size ratio calculated for the case $\beta$ $=1$ forms an upper bound for the realized asymptotic size ratio for cases with $\beta<1$.

\section{Catch-up growth}

The model discussed in the previous section can produce significant growth compensation, and we wish to know if this would allow the growth trajectory of a starved and re-alimented individual to overtake that of one fed continuously. To address this question we consider an experiment in which two individuals start from the state $\left(S_{0}, X^{*}\right)$ at $t=0$. One, whose structure at time $t$ we denote by $S^{*}(t)$, grows normally in the experimental environment. The other, whose structure at time $t$ we denote by $S(t)$, is starved until its reserve fraction reaches a value $X_{0}$, when it is re-introduced to the experimental environment.

In the special case $\beta=1$ we can determine what happens analytically. From Eq. 9 we see that the time, $\tau$, required for the starved individual to reach $X=X_{0}$ is

$$
\tau=\frac{1}{\mu} \ln \left[\frac{1-X_{0}}{1-X^{*}}\right] .
$$

During this time the control individual will have been growing exponentially at the quasi-steady-state rate, so at time $\tau$ the ratio of the structural carbon masses of the starved and control individuals, which we shall call the "deficit ratio" $\left(D_{\tau}\right)$, will be given by

$$
D_{\tau} \equiv \frac{S(\tau)}{S^{*}(\tau)}=\exp \left(-\frac{p k^{*} \alpha X_{\mathrm{d}}}{X^{*}} \tau\right)
$$

where $k^{*}$ represents the proportion of the assimilated protein allocated to structure in the quasi-stationary state. Substituting from Eq. 25 allows us to rewrite this as

$$
D_{\tau}=\left[\frac{1-X_{0}}{1-X^{*}}\right]^{-\left[\left(p k^{*} \alpha X_{\mathrm{d}}\right) /\left(\mu X^{*}\right)\right] .}
$$

Overcompensation requires that that the asymptotic size of the manipulated individual is greater than that of an unmanipulated control. For this to be true, the product of the deficit ratio $\left(D_{\tau}\right.$, Eq. 27) and the asymptotic compensation ratio produced by compensation following re-alimentation at time $\tau\left(C_{\infty}\right.$, Eq. 23) must be greater than 1 .

To see if this is so for the constant-allocation model we recognize from the previous section that for this model variant, $k^{*}=k_{0}$. We then substitute for $X^{*}$ from Eq. 20, and hence find that the deficit ratio is

$$
D_{\tau}=\left[\frac{1-X_{0}}{1-X^{*}}\right]^{-\left[p k_{0} /\left(1-p k_{0}\right)\right]\left[\left(\alpha X_{\mathrm{d}}+\mu\right) / \mu\right]} .
$$

Comparison with Eq. 23 immediately shows that with constant allocation all possible parameter values result in undercompensation.

\section{Net production allocation}

We now consider a model variant in which the hyperphagia factor is still given by Eq. 18 but the fractional allocation of protein to structure $(k)$ is controlled so as to make $X^{*}$ as nearly constant as possible (Eqs. 12 and 13). As we showed earlier, when such control is successful it results in the allocation of a fixed fraction of net production to structural mass.

Substituting Eqs. 12, 17, and 18 into Eq. 8 allows us to show that the quasi-stationary reserve fraction for this model variant is

$$
X^{*}= \begin{cases}\frac{X_{\mathrm{d}}}{X_{\mathrm{d}}+\mu S / \alpha S^{\beta}} & \text { if } \frac{\mu S}{\alpha S^{\beta}}>1-X_{\mathrm{d}} \\ X_{\mathrm{d}} & \text { if } 1-X_{\mathrm{d}} \geq \frac{\mu S}{\alpha S^{\beta}}>1-X_{\mathrm{d}}-p \\ \frac{(1-p) X_{\mathrm{d}}}{X_{\mathrm{d}}+\mu S / \alpha S^{\beta}} & \text { if } 1-X_{\mathrm{d}}-p \geq \frac{\mu S}{\alpha S^{\beta}}>0 .\end{cases}
$$

Since this model variant incorporates a hyperphagic 
response (Eq. 18) it must predict a period of compensatory growth following any series of events that leads to the reserve fraction being depressed below the current steady-state value. In the special case of assimilation that is linear in $S(\beta=1)$, we can calculate the size of the resulting growth increment. We restrict ourselves to values of $\mu / \alpha$ in the range $1-X_{\mathrm{d}} \geq \mu / \alpha>$ $1-X_{\mathrm{d}}-p$, so that $X^{*}=X_{\mathrm{d}}$, and to values of $X_{0}>1$ $-X_{\mathrm{d}}-p$ so that $k$ is always given by Eq. 12. In the Appendix we show that if the control individual starts with structure $S_{0}$ and reserve ratio $X_{\mathrm{d}}$ and the experimental individual starts with structure $S_{0}$ and reserve ratio $X_{0}$, then the asymptotic compensation ratio, $C_{\infty}$, is

$$
C_{\infty} \equiv \lim _{t \rightarrow \infty}\left[\frac{S(t)}{S^{*}(t)}\right]=\left[\frac{1-X_{0}}{1-X_{\mathrm{d}}}\right]^{\left(1-X_{\mathrm{d}}\right) / X_{\mathrm{d}}} .
$$

To see if this asymptotic compensation ratio is large enough to imply overcompensation we return to Eq. 27. Remembering that in the quasi-steady state the reserve ratio is $X_{\mathrm{d}}$ and the allocation fraction is $k^{*}=$ $p^{-1}\left(1-X_{\mathrm{d}}-\mu / \alpha\right)$, we see that the deficit ratio for this case is

$$
D_{\tau}=\left[\frac{1-X_{0}}{1-X_{\mathrm{d}}}\right]^{-\alpha / \mu\left[1-X_{\mathrm{d}}-(\mu / \alpha)\right]} .
$$

Hence we see that compensation following a period of starvation exceeds the growth lost during the starvation period providing that

$$
\frac{X_{\mathrm{d}}\left(1-X_{\mathrm{d}}\right)}{\mu / \alpha}<1
$$

Fig. 5 contrasts individuals with hyperphagic responses given by Eq. 18 and who allocate a fixed fraction of ingested protein to structure (Fig. 5c) against those with the same hyperphagic response but with feeding-rate dependent protein allocation designed to hold $X^{*}$ constant (Fig. 5a). The other parameters are chosen so that an individual with feeding-rate-dependent allocation and $\beta=1$ exhibits over-compensation (Inequality 32). Comparing the trajectories shown in Fig. 5a and $\mathrm{c}$ shows us that it does so because the extra protein it ingests during the period of hyperphagia is routed preferentially to forming new structure. This increases the rate of structural growth at a given value of reserve fraction and decreases the rate of reserve recovery, thus prolonging the hyperphagic period.

In Fig. 5b we show an equivalent simulation for an individual with $\beta=0.5$, which shows an asyptotic size ratio very similar to, but slightly smaller than, that observed with $\beta=1$. We observe this relationship in all our simulations and conclude that Inequality 32 provides a bounding value in the sense that a parameter set that would compensate incompletely with $\beta=1$ will behave similarly with $\beta<1$.
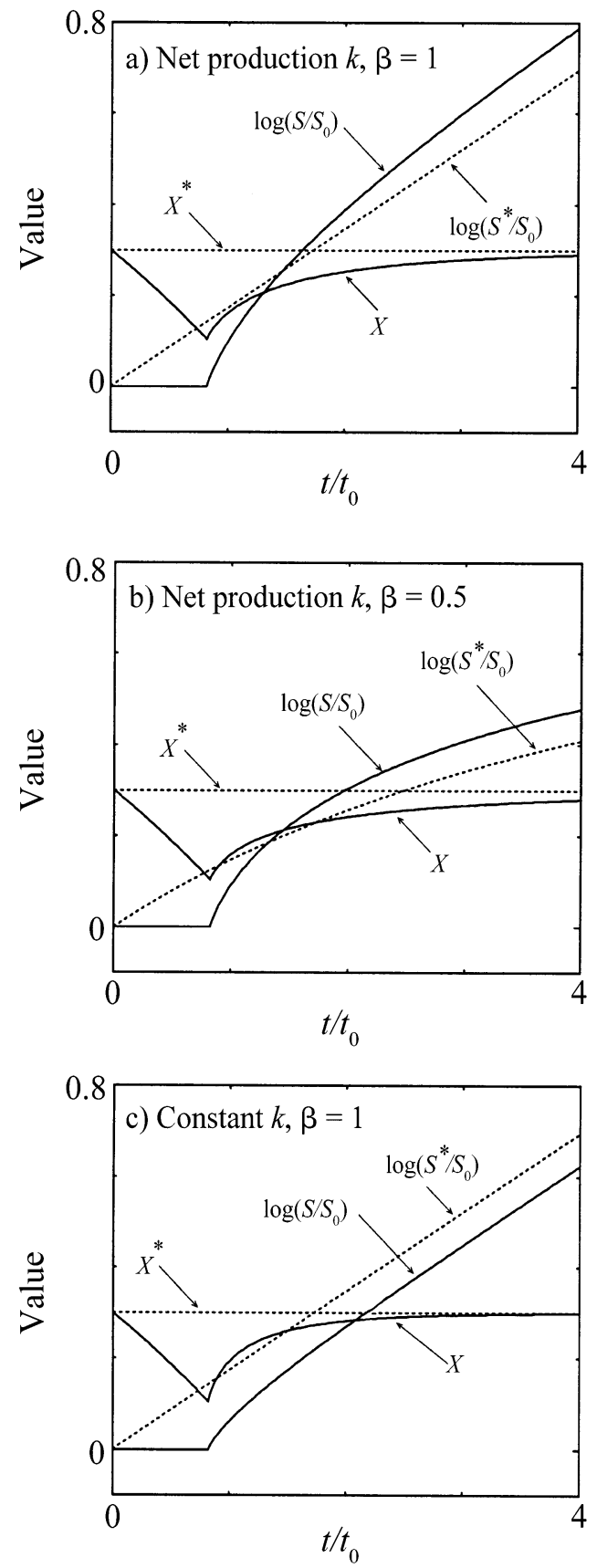

FIG. 5. Catch-up growth. All panels compare the performance of two initially identical individuals with hyperphagic response defined by Eq. 18 raised in the experimental environment. One (dotted lines) grows continuously in the experimental environment, while the other (solid lines) is starved until its reserve fraction reaches one-third of the quasi-stationary value and thereafter grows in the experimental environment. Structure and time scales are $S_{0}=S(0)$ and $t_{0}$ $=1 /\left(\alpha S_{0}^{\beta-1}\right)$, respectively. Common parameters are $\mu t_{0}=0.3$, $X_{\mathrm{d}}=0.3, p=0.8$. Panels (a) and (b) present solutions with $k$ controlled so as to hold $X^{*}=X_{\mathrm{d}}$ (Eq. 13). (c) An equivalent solution with $k$ held constant at the steady-state value (0.5). 


\section{Discussion}

\section{Diet and allocation}

The aim of this paper is to understand the way in which the mechanisms controlling hyperphagia and resource allocation interact to determine the compensatory growth response. We have examined a series of strategic energy-budget models, which describe individual state in terms of nonmetabolizable (structural) carbon mass $(S)$ and the metabolizable (reserve) fraction of total carbon mass $(X)$. In a constant environment, the reserve fraction of an individual whose assimilation rate $A$ scales linearly with $S$ tends to to a size-independent steady-state value, $X^{*}$. When $A$ varies allometrically with $S$, the analogous quasi-steady state varies weakly with $S$, but still provides a good longterm estimate of $X$.

Our modeling framework divides net assimilate into two components, one of which ("fat") can only be used to meet current or future metabolic costs, while the other ("protein") can be used either to meet metabolic costs or to form new structural mass. When the proportion of protein in the assimilate $(p)$ and the proportion of assimilated protein allocated to structure $(k)$ are both constant, the individual can only establish a positive stationary (or quasi-stationary) reserve fraction if the total flow of carbon to reserves and maintenance $(A(1-k p))$ exceeds the cost of maintaining its structural mass. At lower assimilation rates new structure is still produced, but reserves are burned to meet basal metabolic costs - a policy that eventually results in reserve exhaustion and death.

Such anomalies are avoided by varying the protein allocation proportion $k$. In the model proposed by Jones et al. (2002) this happens in response to low reserve fraction, whereas in the model presented here $k$ is controlled directly by feeding rate. We have shown that where feeding-rate-controlled allocation succeeds in making $X^{*}$ independent of $A$, a fixed fraction of net production is allocated to the creation of new structural mass. A similar result can be derived for conditioncontrolled allocation.

\section{Hyperphagia and compensation}

Our representation of compensatory hyperphagia assumes that in a constant environment the individual's net assimilation rate varies inversely with reserve ratio. Although this formulation permits unlimited (and clearly unrealistic) appetite increases as the reserve ratio goes to zero, the payoff is that for assimilation that is linear in $S$ we can obtain an analytic expression for the total compensation exhibited by an individual with constant fractional protein allocation $(k)$. Our numerical experiments show that this expression also forms an upper bound when assimilation scales sublinearly with $S$ and when the appetite is limited to a realistic multiple of that at the quasi-stationary state.
To determine whether compensatory hyperphagia can enable a starved and re-fed individual to catch or outgrow one fed continuously, we calculated the growth increment produced by an individual whose assimilation is linear in $S$, growing continuously at the steady-state reserve ratio for the time taken to starve an initially identical individual to some given reserve fraction. We then compared the result with the compensatory growth increment produced when the starved individual is re-fed.

For individuals with linear assimilation allometry whose allocation of assimilated protein to structure is held constant, we were able to show that no combination of parameters would enable the starved and refed individual to equal, still less surpass, the growth of its continuously fed equivalent. Since we believe that our analytic result for linear assimilation allometry and unlimited appetite is an upper bound for more realistic descriptions (sublinear assimilation allometry and/or limited appetite) we conclude that compensatory hyperphagia acting alone and in inverse proportion to reserve fraction cannot generate overcompensation or even complete catch-up.

Concerns that this result might be an artifact of our hypothesized relation between hyperphagia and reserve fraction led us to conduct a series of numerical experiments with other functional forms, paying particular attention to those in which appetite is more sensitive to $X$. Although such formulations can lead to increased compensation when unlimited appetite increases are permitted, their performance differs little from that of our primary description when appetite increases are restricted to one or two orders of magnitude above normal. We thus conclude that hyperphagia acting alone cannot generate the strong compensation required for full growth catch-up or even overcompensation.

The reason for this outcome is that with constant allocation of protein to structure, the compensatory increase in assimilation rapidly restores the reserve fraction to its stationary value, thus terminating the period of hyperphagia. Jobling and Johansen (1999) have pointed out that preferential allocation of ingested protein to structure during hyperphagia prolongs the hyperphagic period and thus enhances the total compensatory response.

A control mechanism with just this effect is the one we introduced to render the steady-state reserve fraction independent of assimilation rate, which it achieves by increasing allocation to structure at high assimilation rates and decreasing it at low assimilation rates. A model combining this allocation control mechanism with compensatory hyperphagia shows characteristically increased hyperphagic periods and strengthened compensatory response.

When assimilation is linear in $S$, we can again calculate both the compensatory growth increment and the increment needed for full catch-up, thus obtaining an inequality that allows us to define the conditions 
required for overcompensation. This shows that overcompensation is more likely when the assimilation rate scale $(\alpha)$ is not too far in excess of the basal metabolic rate scale $(\mu)$. Indeed, when $\mu / \alpha>0.25$, the overcompensation requirement is satisfied for all values of the steady-state reserve fraction, $X_{\mathrm{d}}$.

\section{Conclusions}

We conclude that hyperphagia acting alone cannot generate a sufficiently strong compensatory response to allow the growth of a starved and re-fed individual to equal, still less surpass, that of a continuously fed equivalent. However, when combined with a mechanism that adjusts protein allocation between reserves and structure in response to changes in assimilation rate in such a way as to increase allocation to structure at high feeding rates, complete catch-up can occur over a wide range of parameters.

The effect of the required allocation-control mechanism is to reduce the dependence of the (quasi-) steady-state reserve fraction on assimilation rate. If this dependency can be entirely removed, the effect is to cause a fixed fraction of somatic production (net of basal metabolic costs) to be allocated to the generation of new structure.

Models of the class discussed in this paper are qualitatively capable of describing all observed patterns of compensatory growth following a change in environment that results in an increase in the steady-state reserve fraction. Such changes clearly include the reappearance of food after a period of starvation, for example a spring phytoplankton bloom or a spring flush in stream invertebrates. The extent to which other changes, such as a rapid increase in food availability or temperature, will generate compensation depends on the extent to which the steady-state reserve fraction depends on the environment.

Experiments in which an individual is kept on at maintenance ration raise particularly subtle issues. If the experimenter calculates the maintenance ration so as to exactly stop structural growth without causing any reduction in total carbon mass, i.e., the ingestion rate exactly matches the current basal metabolic cost, then reintroduction to abundant food will cause no compensatory response. However, if the experimenter simply succeeds in the more plausible goal of setting a ration at which the individual does not die (that is, a ration that exceeds the basal metabolic cost of the animal's current structure), then a strong compensatory response can be expected. It seems possible that such considerations explain the wide variability in results from experiments involving periods at a maintenance ration.

\section{ACKNOWLEDGMENTS}

This work was supported by the NERC through a research studentship to W. Jones and through partial support under research grant NER/T/S/1999/00072. We thank Graeme Ruxton, Neil Metcalfe, and Malcolm Elliott for helpful conversations about energy allocation in juvenile salmonids.

\section{Literature Cited}

Blum, W. F. 1997. Leptin: the voice of the adipose tissue. Hormone Research 48:2-8.

Bradley, M. C., N. Perrin, and P. Calow. 1991. Energy allocation in the clodoceran Daphnia magna Straus under starvation and refeeding. Oecologia 86:414-418.

Broekhuisen, N. W., W. S. C. Gurney, A. Jones, and A. Bryant. 1994. Modelling compensatory growth. Functional Ecology 8:770-782.

Bull, C. D., and N. B. Metcalfe. 1996. Regulation of hyperphagia in response to varying energy deficits in over-wintering juvenile Atlantic salmon. Journal of Fish Biology 20:498-510.

Carter, C. G., D. F. Houlihan, and I. D. McCarthy. 1992. Feed utilisation efficiencies of Atlantic salmon (Salmo salar) Parr: effect of single supplementary enzyme. Comparative Biochemistry \& Physiology A 101:369-374.

Friedman, J. M. 1998. Leptin, leptin receptors and the control of body weight. Nutritional Reviews 56:38-46.

Gurney, W. S. C., and R. M. Nisbet. 1998. Ecological dynamics. Oxford University Press, New York, New York, USA.

Hayward, R. S., D. B. Noltie, and N. Wang. 1997. Use of compensatory growth to double hybrid sunfish growth rates. Transactions of the American Fisheries Society 126: 316-322.

Jobling, M., and S. J. S. Johansen. 1999. The lipostat, hyperphagia and catch-up growth. Aquaculture Research. 30: 473-478.

Jones, W. 2001. Modelling the growth and resource allocation dynamics of juvenile salmonids. Dissertation. University of Strathclyde, Glasgow, Scotland.

Jones, W., W. S. C. Gurney, D. C. Speirs, P. J. Bacon, and A. F. Youngson. 2002. Seasonal patterns of growth, expenditure and assimilation in juvenile Atlantic salmon. Journal of Animal Ecology 71:916-924.

Kennedy, G. C. 1953. The role of depot fat in hypothalmic control of food intake in the rat. Proceedings of the Royal Society B 140:578-592.

Kooijman, S. A. L. M. 1993. Dynamic energy budgets in biological systems. Cambridge University Press, Cambridge, UK.

McCauley, E., W. W. Murdoch, R. M. Nisbet, and W. Gurney. 1990. The physiological ecology of Daphnia: development of a model of growth and reproduction. Ecology 71:703715 .

Metcalfe, N. B., and J. E. Thorpe. 1992. Anorexia and defended energy levels in over-wintering juvenile salmon. Journal of Animal Ecology 61:175-181.

Miglavs, I., and M. Jobling. 1989a. Effects of feeding regime on food consumption, growth rates and tissue nucleic acids in juvenile Arctic charr, Salvelinus alpinus, with particular reference to compensatory growth. Journal of Fish Biology 34:997-957.

Miglavs, I., and M. Jobling. 1989b. The effects of feeding regime on proximate body composition and patterns of energy deposition in juvenile Arctic charr, Salvelinus alpinus. Journal of Fish Biology 35:1-11.

Muller, E. B. and R. M. Nisbet. 2000. Survival and production in variable resource environments. Bulletin of Mathematical Biology 62:1163-1189.

Nisbet, R. M., E. B. Muller, K. Lika, and B. Kooijman. 2000. From molecules to ecosystems through dynamic energy budget models. Journal of Animal Ecology 69:913-926.

O'Connor, K. I., A. C. Taylor, and N. B. Metcalfe. 2000. The stability of standard metabolic rate during a period of food deprivation in juvenile Atlantic salmon. Journal of Fish Biology 57:41-51.

Russel, N. R., and R. J. Wooton. 1992. Appetite and growth compensation in the European minnow, Phoxinus phoxinus, 
following short periods of food restriction. Environmental Biology of Fishes 34:277-285.

Weatherly, A. H., and R. W. Gill. 1981. Recovery growth following periods of restricted rations and starvation in rainbow trout Salmo gairdneri Richardson. Journal of Fish Biology 18:195-208.

Weigle, D. S. 1994. Appetite and the regulation of body composition. FASEB Journal 8:302-310.
Wilson, P. N., and D. F. Osbourne. 1960. Compensatory growth after under-nutrition in mammals and birds. Biological Reviews of the Cambridge Philo-sophical Society 37:324-363.

Yu, M. W., F. E. Robinson, M. T. Clandinin, and L. Bodnar 1990. Growth and body-composition of broiler chickens in response to different regimes of feed restriction. Poultry Science 69:2074-2081.

\section{APPENDIX}

\section{Asymptotic Size Ratio with $\beta=1$}

Constant $k$

Consider two solutions of Eqs. 19 and 21 with $\beta=1$. The first solution, $\left(S^{*}(t), X^{*}\right)$, starts from the initial condition $\left(S_{0}\right.$, $\left.X^{*}\right)$, while the second, $(S(t), X(t))$, starts from the initial condition $\left(S_{0}, X_{0}\right)$.

Eq. 21 tells us that

$$
\ln \left[\frac{S(t)}{S^{*}(t)}\right]=\frac{p k_{0} \alpha X_{\mathrm{d}}}{X^{*}} \int_{0}^{t}\left(\frac{X^{*}-X}{X}\right) d t .
$$

Eqs. 19 and 20 show that this can be rewritten as

$$
\ln \left[\frac{S(t)}{S^{*}(t)}\right]=\frac{p k_{0}}{1-p k_{0}} \int_{X_{0}}^{X} \frac{d X}{1-X} .
$$

Integrating Eq. A.2 and remembering that, so long as $X^{*}$ $>0$, then $X \rightarrow X^{*}$ as $t \rightarrow \infty$, then

$$
\lim _{t \rightarrow \infty}\left[\frac{S(t)}{S^{*}(t)}\right]=\left[\frac{1-X_{0}}{1-X^{*}}\right]^{-p k_{0} /\left(1-p k_{0}\right)} .
$$

"Net-production" $k$

Consider two solutions of Eqs. 8 and 6 with A given by Eq. $17, \lambda$ given by Eq. $18, k$ given by Eq. 13 , and $\beta=1$.
We confine ourselves to values of $\mu / \alpha$ in the range $\left[1-X_{\mathrm{d}}\right.$ $-p, 1-X_{\mathrm{d}}$ ], so that the quasi-stationary reserve fraction $X^{*}$ $=X_{\mathrm{d}}$ (Eq. 29).

The first solution, $\left(S^{*}(t), X_{\mathrm{d}}\right)$, starts from the initial condition $\left(S_{0}, X_{\mathrm{d}}\right)$, while the second, $(S(t), X(t))$, starts from the initial condition $\left(S_{0}, X_{0}\right)$. We confine ourselves to values of $X_{0}>1-X_{\mathrm{d}}-p$, so that $k$ is always given by Eq. 12 .

Eq. 6 together with Eqs. 17, 18, 13, and 29, tells us that

$$
\ln \left[\frac{S(t)}{S^{*}(t)}\right]=\frac{\alpha X_{\mathrm{d}}\left(1-X_{\mathrm{d}}\right)}{X_{\mathrm{d}}} \int_{0}^{t}\left(\frac{X_{\mathrm{d}}-X}{X}\right) d t .
$$

Eq. 8 together with Eqs. 17, 18, 13, and 29, shows that this can be rewritten as

$$
\ln \left[\frac{S(t)}{S^{*}(t)}\right]=\frac{1-X_{\mathrm{d}}}{X_{\mathrm{d}}} \int_{X_{0}}^{X} \frac{d X}{1-X} .
$$

Integrating Eq. A.5 and remembering that $X \rightarrow X_{\mathrm{d}}$ as $t \rightarrow$ $\infty$, then shows that

$$
\lim _{t \rightarrow \infty}\left[\frac{S(t)}{S^{*}(t)}\right]=\left[\frac{1-X_{0}}{1-X_{\mathrm{d}}}\right]^{\left(1-X_{\mathrm{d}}\right) / X_{\mathrm{d}}} .
$$

